Jean Bertoin

\title{
A second order SDE for the Langevin process reflected at a completely inelastic boundary
}

Received October 14, 2006 and in revised form December 22, 2006

Abstract. It was shown in [2] that a Langevin process can be reflected at an energy absorbing boundary. Here, we establish that the law of this reflecting process can be characterized as the unique weak solution to a certain second order stochastic differential equation with constraints, which is in sharp contrast with a deterministic analog.

Keywords. Langevin process, reflection, stochastic differential equation

\section{Introduction}

Consider the motion of a particle in the half-line $\mathbb{R}_{+}$under an external force that governs its acceleration. Assume that the energy of the particle is instantaneously absorbed at the boundary point 0 , meaning that the velocity of the particle is always 0 immediately after hitting 0 . In other words, the trajectory $\left(X_{t}\right)_{t \geq 0}$ of the particle fulfills the constraints of completely inelastic impacts

$$
\left\{\begin{array}{l}
X_{t} \geq 0 \\
X_{t}=0 \Rightarrow \dot{X}_{t+}=0,
\end{array}\right.
$$

and solves the second order differential equation

$$
d X_{t}=\dot{X}_{t} d t, \quad d \dot{X}_{t}=F_{t} d t+d A_{t}, \quad A_{t}=-\sum_{0<s \leq t} \dot{X}_{s-} \mathbf{1}_{\left\{X_{s}=0\right\}},
$$

where $\left(F_{t}\right)_{t \geq 0}$ denotes the external force. More precisely, $t \mapsto A_{t}$ is right-continuous non-decreasing and accounts for the kick induced by the boundary. Specifically, if the particle hits 0 at time $t$ with incoming velocity $\dot{X}_{t-}<0$, then $A_{t}-A_{t-}=-\dot{X}_{t-}>0$ so that $\dot{X}_{t+}=\dot{X}_{t-}+\left(A_{t}-A_{t-}\right)=0$.

Equation (2) can be viewed as a special case of differential measure inclusions which have been studied initially by Schatzman [13]; see also Ballard [1] and the references therein. It is quite remarkable that multiple solutions may exist even in situations when the external force is $\mathcal{C}^{\infty}$.

J. Bertoin: Laboratoire de Probabilités et Modèles Aléatoires, Université Pierre et Marie Curie, and DMA, École Normale Supérieure, Paris, France; e-mail: jean.bertoin@upmc.fr

Mathematics Subject Classification (2000): Primary 60H10, 60J55; Secondary 34A12 
Following a question raised by Bertrand Maury, we are interested in the case when the external force is a generalized function given by a white noise, i.e. when $F_{t}=\dot{B}_{t}$ with $\left(B_{t}\right)_{t \geq 0}$ a standard Brownian motion. In this setting, it is natural to consider first the much simpler situation when there is no obstacle at 0 , that is, to introduce the process with values in $\mathbb{R}$

$$
Y_{t}=y_{0}+t \dot{y}_{0}+\int_{0}^{t} B_{s} d s, \quad t \geq 0 .
$$

The latter will be called here a free Langevin process, starting from $y_{0} \in \mathbb{R}$ and with initial velocity $\dot{y}_{0} \in \mathbb{R}$; we refer to Lachal [5] for a rich source of results and references in this area. It is easily seen that for $y_{0}=\dot{y}_{0}=0,0$ is an accumulation point of the set of times at which the free Langevin process returns to 0 . Informally, this may suggest that if the energy of the Langevin particle is absorbed at each visit to 0 , then the particle might never be able to reach a strictly positive velocity, and thus might never take off the boundary. It turns out that this intuition is incorrect.

It is convenient to agree that throughout this work, all random processes are implicitly càdlàg, i.e. their sample paths are right-continuous and have left limits everywhere, a.s. In a preceding work [2], we established the following result of existence and uniqueness in distribution.

Theorem 1. There exists a strong Markov process $\left(X_{t}, \dot{X}_{t}\right)_{t \geq 0}$ with values in $\mathbb{R}_{+} \times \mathbb{R}$, starting from $X_{0}=\dot{X}_{0}=0$, such that

$$
d X_{t}=\dot{X}_{t} d t, \quad \int_{0}^{\infty} \mathbf{1}_{\left\{X_{t}=0\right\}} d t=0 \text { and } X_{t}=0 \Rightarrow \dot{X}_{t}=0 \quad \text { a.s. }
$$

and which evolves as a free Langevin process as long as $X>0$. Specifically, for every stopping time $S$ in the natural filtration of $X$ (after the usual completions), if we define $\zeta_{S}=\inf \left\{t \geq 0: X_{S+t}=0\right\}$, then conditionally on $X_{S}=x_{0}>0$ and $\dot{X}_{S}=\dot{x}_{0}$, the process $\left(X_{S+t}\right)_{0 \leq t \leq \zeta_{S}}$ is independent of $\mathcal{F}_{S}$ and has the same distribution as $\left(Y_{t}\right)_{0 \leq t \leq \zeta}$, where

$$
Y_{t}=x_{0}+t \dot{x}_{0}+\int_{0}^{t} B_{s} d s, \quad \zeta=\inf \left\{t \geq 0: Y_{t}=0\right\}
$$

and $\left(B_{t}\right)_{t \geq 0}$ is a standard Brownian motion. Furthermore, the preceding requirements determine the distribution of $\left(X_{t}, \dot{X}_{t}\right)_{t \geq 0}$.

We stress that the strong Markov process $\left(X_{t}, \dot{X}_{t}\right)_{t \geq 0}$ has jumps at predictable stopping times (namely, the hitting times by $X$ of the boundary point 0 ), and thus fails to be standard; in particular, the Feller property does not hold.

The main purpose of this work is to connect the process characterized in Theorem 1 to equation (2) when the external force $F$ is a white noise. With this in view, it is convenient to rewrite (2) in the form

$$
d X_{t}=\dot{X}_{t} d t, \quad \dot{X}_{t}=B_{t}+A_{t}, \quad A_{t}=-\sum_{0<s \leq t} \dot{X}_{s-} \mathbf{1}_{\left\{X_{s}=0\right\}} .
$$

We are now able to state the main result of this work. 
Theorem 2. (i) One can construct on some filtered probability space $\left(\Omega,\left(\mathcal{F}_{t}\right)_{t \geq 0}, \mathbb{P}\right)$ an adapted process $\left(X_{t}\right)_{t \geq 0}$ distributed as in Theorem 1 and an $\left(\mathcal{F}_{t}\right)$-Brownian motion $\left(B_{t}\right)_{t \geq 0}$ such that equations (1) and (4) hold.

(ii) Conversely, if on some filtered probability space $\left(\Omega,\left(\mathcal{F}_{t}\right)_{t \geq 0}, \mathbb{P}\right)$, there is an $\left(\mathcal{F}_{t}\right)$ Brownian motion $\left(B_{t}\right)_{t \geq 0}$ and an adapted process $\left(X_{t}\right)_{t \geq 0}$ which satisfies equations (1) and (4) and starts with initial conditions $X_{0}=\dot{X}_{0}=0$, then $\left(X_{t}\right)_{t \geq 0}$ is distributed as in Theorem 1.

We shall refer to the process $X$ which appears in Theorems 1 and 2 as a Langevin process reflected at a completely inelastic boundary. Note that we implicitly restrict our attention to the case when the process starts from 0 with initial velocity 0 , which is obviously the most interesting situation and involves no loss of generality. In some loose sense, Theorems 1 and 2 both state the existence and uniqueness in law of the Langevin process reflected at a completely inelastic boundary, but viewed from two different perspectives. Theorem 1 belongs to the framework of the theory of Markov processes and their excursions, whereas Theorem 2 is expressed in terms of stochastic differential equations. It is well-known that these two theories are intimately connected, and one can expect that a soft argument should enable us to deduce Theorem 2 from Theorem 1.

In this direction, the existence of a weak solution to (4) and (1) is rather easy and will be established in the first part of Section 2 by investigating, in the framework of stochastic calculus, the explicit construction given in [2] of the process specified by Theorem 1 More precisely, the latter is obtained from the free Langevin process associated to some standard Brownian motion $\left(W_{t}\right)_{t \geq 0}$ first by a reflection à la Skorokhod and then by a noninvertible random time-substitution.

However, establishing weak uniqueness in Theorem 2 is less straightforward. Indeed, if we aim at applying Theorem 1 , then we have to check a priori that any weak solution $\left(X_{t}, \dot{X}_{t}\right)_{t \geq 0}$ to (4) and (1) enjoys the strong Markov property. But it is well-known that solutions of an SDE have the Markov property only when weak uniqueness holds for the SDE, and thus Theorem 1 cannot help. We also stress that weak uniqueness is the most striking aspect of Theorem 2 as it is in sharp contrast with the deterministic situation for which (strong) uniqueness can fail even with a smooth forcing.

In the second part of Section 2, we shall observe a key point which lies at the heart of the proof of weak uniqueness. From the same Brownian motion $\left(W_{t}\right)_{t \geq 0}$ which is used to construct a weak solution $\left(X_{t}, B_{t}\right)_{t \geq 0}$, one can also build another standard Brownian motion $\left(B_{t}^{\prime}\right)_{t \geq 0}$ which is independent of $\left(B_{t}\right)_{t \geq 0}$, and such that $\left(W_{t}\right)_{t \geq 0}$ can be recovered from $\left(X_{t}, B_{t}\right)_{t \geq 0}$ and $\left(B_{t}^{\prime}\right)_{t \geq 0}$. Weak uniqueness is established in Section 3. We consider any solution $\left(X_{t}, B_{t}\right)_{t \geq 0}$ to (4) and (1) where $\left(B_{t}\right)_{t \geq 0}$ is some Brownian motion. We then introduce an independent standard Brownian motion $\left(B_{t}^{\prime}\right)_{t \geq 0}$, and using the analysis developed in Section 2, we construct from $\left(X_{t}, B_{t}\right)_{t \geq 0}$ and $\left(B_{t}^{\prime}\right)_{t \geq 0}$ another Brownian motion $\left(W_{t}\right)_{t \geq 0}$ such that $\left(X_{t}\right)_{t \geq 0}$ can be recovered from $\left(W_{t}\right)_{t \geq 0}$ in the same way as in Section 2.

In the final section, we first make some brief historical comments about the question of uniqueness in the-deterministic - setting of mechanical systems with perfect constraints. For the reader's convenience, we also provide a simple example showing that 
uniqueness of the solution to (1) and (2) may fail even when the external force is smooth. Finally, we discuss some open questions regarding strong solutions to (4) and (1).

Nota Bene. In this paper, I will use the same notation $X, B, W, \ldots$ for processes which, in fine, will be shown to have the same distributions. However the initial definition and assumptions for these processes may be different in different sections. I hope that the reader will find this helpful and not confusing.

\section{A weak solution}

The first purpose of this section is to check that the construction of Section 2 in [2] also provides a solution to (4) and (1). Then we shall study this construction in further details to gain insight for the proof of weak uniqueness.

\subsection{Construction of a weak solution}

We start by recalling the construction of Section 2 in [2] and some of its properties.

Let $W=\left(W_{t}, t \geq 0\right)$ be a standard Wiener process starting from $W_{0}=0$ and write $\left(\mathcal{W}_{t}\right)_{t \geq 0}$ for its natural filtration after the usual completions. Define the free Langevin process

its infimum process

$$
Y_{t}:=\int_{0}^{t} W_{s} d s, \quad t \geq 0,
$$

$$
I_{t}:=\inf \left\{Y_{s}: 0 \leq s \leq t\right\},
$$

and the random closed set of times when $Y$ coincides with its infimum,

$$
\mathcal{I}:=\left\{t \geq 0: Y_{t}=I_{t}\right\} \text {. }
$$

We write $\mathcal{I}^{\circ}$ for the interior of $\mathcal{I}$ and recall from Lemma 2 in [2] that with probability one, the boundary $\partial \mathcal{I}=\mathcal{I} \backslash \mathcal{I}^{\circ}$ has zero Lebesgue measure. Further, the canonical decomposition of the open set $\mathcal{I}^{\circ}$ into disjoint open intervals is given by

$$
\left.\mathcal{I}^{\circ}=\bigcup_{u \in \mathcal{J}}\right] u, d_{u}[\text {, }
$$

where $\mathcal{J}$ is the set of times at which $Y$ reaches its infimum for the first time during some negative excursion of $W$, and $d_{u}$ the first return time to 0 for $W$ after the instant $u$. That is,

$$
\mathcal{J}:=\left\{t \geq 0: W_{t}<0, Y_{t}=I_{t} \text { and } Y_{t-\varepsilon}>I_{t-\varepsilon} \text { for all } \varepsilon>0 \text { sufficiently small }\right\}
$$

and

$$
d_{u}:=\inf \left\{s>u: W_{s}=0\right\} .
$$

It is readily seen that $\mathcal{J}$ can be expressed in the form of a countable family of stopping times in the filtration $\left(\mathcal{W}_{t}\right)_{t \geq 0}$. For instance $\mathcal{J}=\left\{S_{k, n}: k, n \in \mathbb{N}\right\}$, where $S_{k, n}$ is the $k$-th instant $t$ such that $Y_{t}=I_{t}, Y_{t-\varepsilon}>I_{t-\varepsilon}$ for all $\varepsilon>0$ sufficiently small and the velocity at time $t$ satisfies $\left.\left.\dot{Y}_{t} \in\right]-1 /(n-1),-1 / n\right]$. Note that the $d_{S_{k, n}}$ are then also stopping times. 
Finally, we introduce the right-continuous time-substitution

$$
T_{t}:=\inf \left\{s \geq 0: \int_{0}^{s} \mathbf{1}_{\left\{Y_{v}>I_{v}\right\}} d v>t\right\}, \quad t \geq 0,
$$

and then the free Langevin process reflected at its infimum (in the sense of Skorokhod) and time-changed by $T_{t}$, that is, for every $t \geq 0$ we set

$$
X_{t}:=(Y-I) \circ T_{t}
$$

We mention that the process $t \mapsto Y_{t}-I_{t}$ has been studied first by Lapeyre [6]. Clearly, the process $t \mapsto X_{t}$ only takes nonnegative values, is continuous, and it can be shown that it has a right derivative at every $t \geq 0$, given by

$$
\dot{X}_{t}=W \circ T_{t} ;
$$

see equation (7) in [2]. We also set $\mathcal{F}_{t}=\mathcal{W}_{T_{t}}$.

The following proposition establishes the existence stated in Theorem 2 (i).

Proposition 1. Define

$$
A_{t}:=\int_{0}^{T_{t}} \mathbf{1}_{\left\{Y_{s}=I_{s}\right\}} d W_{s} \quad \text { and } \quad B_{t}:=\int_{0}^{T_{t}} \mathbf{1}_{\left\{Y_{s}>I_{s}\right\}} d W_{s}, \quad t \geq 0,
$$

so that

$$
\dot{X}_{t}=A_{t}+B_{t}, \quad t \geq 0 \text {. }
$$

Then we have the identity

$$
A_{t}=-\sum_{0<s \leq t} \dot{X}_{s-} \mathbf{1}_{\left\{X_{s}=0\right\}}, \quad t \geq 0
$$

and $\left(B_{t}\right)_{t \geq 0}$ is an $\left(\mathcal{F}_{t}\right)$-Brownian motion. As a consequence, $\left(X_{t}, \dot{X}_{t}\right)_{t \geq 0}$ is a weak solution to (4) and (1) with initial condition $X_{0}=\dot{X}_{0}=0$.

Remark. The fact that the series $\sum_{0<s \leq t} \dot{X}_{s-} \mathbf{1}_{\left\{X_{s}=0\right\}}$ converges for every $t \geq 0$ a.s. can be deduced from Corollary 2 in [2]. However, this fact will be established directly in the present analysis.

Proof. We write $\dot{X}_{t}=W \circ T_{t}=A_{t}+B_{t}$, where $A_{t}$ and $B_{t}$ are defined in the statement. The basic facts that have been recalled above imply the identities

$$
\int_{0}^{t} \mathbf{1}_{\left\{Y_{s}=I_{s}\right\}} d W_{s}=\int_{0}^{t} \mathbf{1}_{\{s \in \mathcal{I}\}} d W_{s}=\int_{0}^{t} \mathbf{1}_{\left\{s \in \mathcal{I}^{\circ}\right\}} d W_{s}=\sum_{u \in \mathcal{J}}\left(W_{d_{u} \wedge t}-W_{u \wedge t}\right) .
$$

On the one hand, the assertion that $\left(B_{t}\right)_{t \geq 0}$ is an $\left(\mathcal{F}_{t}\right)$-Brownian motion is seen from the very definition of the time-substitution $T_{t}$ and the Dambis-Dubins-Schwarz theorem 
(see e.g. [12, p. 181]). On the other hand, again by definition, $T_{t} \notin \mathcal{I}^{\circ}$ and $W_{d_{u}}=0$ for every $u \in \mathcal{J}$. We deduce from (5) the identity

$$
A_{t}=-\sum_{u \in \mathcal{J}, u \leq T_{t}} W_{u} .
$$

Further, it is easily checked that $\mathcal{J}$ coincides with the set of times of the form $u=T_{S-}$ with $s>0$ an instant at which $X$ hits the boundary point 0 with a negative incoming velocity (i.e. $X_{s}=0$ and $\dot{X}_{s-}<0$ ). Since $\dot{X}_{s-}=W \circ T_{s-}$, we conclude that

$$
A_{t}=-\sum_{0<s \leq t} \dot{X}_{s-} \mathbf{1}_{\left\{X_{s}=0\right\}}, \quad t \geq 0 .
$$

To complete the proof, either we observe that if $t$ is an instant at which $X_{t}=0$, then $\dot{X}_{t-}=B_{t}+A_{t-}$ and thus

$$
\dot{X}_{t}=B_{t}+A_{t}=\dot{X}_{t-}+\left(A_{t}-A_{t-}\right)=\dot{X}_{t-}-\dot{X}_{t-}=0,
$$

or we just recall from equation (3) in Theorem 1 that $X_{t}=0 \Rightarrow \dot{X}_{t}=0$.

\subsection{Some further properties}

We introduce the time-substitution

$$
T_{t}^{\prime}:=\inf \left\{s \geq 0: \int_{0}^{s} \mathbf{1}_{\left\{Y_{v}=I_{v}\right\}} d v>t\right\}, \quad t \geq 0,
$$

which can be thought of as the dual to $T_{t}$. Next we set

$$
B_{t}^{\prime}:=\int_{0}^{T_{t}^{\prime}} \mathbf{1}_{\left\{Y_{v}=I_{v}\right\}} d W_{v}, \quad t \geq 0,
$$

and then, for every $x \geq 0$,

$$
\sigma^{\prime}(x):=\inf \left\{t \geq 0: B_{t}^{\prime}>x\right\}
$$

for the first passage time of $B^{\prime}$ above level $x$.

Lemma 1. With probability one, we have the identity

$$
T_{t}=t+\sigma^{\prime}\left(A_{t}\right), \quad t \geq 0 .
$$

Proof. It will be convenient to write $B^{\prime}(t):=B_{t}^{\prime}$ and observe from the definition of $B^{\prime}$ and $A_{t}$ (in Proposition 1) the identities

$$
\begin{aligned}
\sigma^{\prime}\left(A_{t}\right) & =\inf \left\{\int_{0}^{s} \mathbf{1}_{\left\{Y_{v}=I_{v}\right\}} d v: B^{\prime}\left(\int_{0}^{s} \mathbf{1}_{\left\{Y_{v}=I_{v}\right\}} d v\right)>A_{t}\right\} \\
& =\inf \left\{\int_{0}^{s} \mathbf{1}_{\left\{Y_{v}=I_{v}\right\}} d v: \int_{0}^{s} \mathbf{1}_{\left\{Y_{v}=I_{v}\right\}} d W_{v}>\int_{0}^{T_{t}} \mathbf{1}_{\left\{Y_{v}=I_{v}\right\}} d W_{v}\right\} .
\end{aligned}
$$

Then recall (5). Observe that for every $u \in \mathcal{J}$, the process $s \mapsto W_{d_{u} \wedge s}-W_{u \wedge s}$ is 0 before time $u$, takes some strictly positive values immediately after time $u$, reaches its overall maximum for the first time at $d_{u}$, and remains constant after $d_{u}$. Note furthermore that the intervals $\left[u, d_{u}\right]$ for $u \in \mathcal{J}$ are pairwise disjoint. It follows that whenever $t \notin \mathcal{I}^{\circ}$, 
the stochastic integral $s \mapsto \int_{0}^{s} \mathbf{1}_{\left\{Y_{v}=I_{v}\right\}} d W_{v}$ attains its overall maximum on the timeinterval $[0, t]$ at time $t$, and if we define $r(t)=\inf \left\{s>t: s \in \mathcal{I}^{\circ}\right\}$, then the first instant when this stochastic integral exceeds its value at time $t$ is $r(t)$. Further, this stochastic integral remains constant on $[t, r(t)]$.

Applying these observations to the random time $T_{t} \notin \mathcal{I}^{\circ}$, we conclude that

$$
\sigma^{\prime}\left(A_{t}\right)=\int_{0}^{r\left(T_{t}\right)} \mathbf{1}_{\left\{Y_{v}=I_{v}\right\}} d v=\int_{0}^{T_{t}} \mathbf{1}_{\left\{Y_{v}=I_{v}\right\}} d v,
$$

and thus

$$
T_{t}=\int_{0}^{T_{t}} \mathbf{1}_{\left\{Y_{v}>I_{v}\right\}} d v+\int_{0}^{T_{t}} \mathbf{1}_{\left\{Y_{v}=I_{v}\right\}} d v=t+\sigma^{\prime}\left(A_{t}\right),
$$

as stated.

We are now able to establish the following statement, which will provide us with the hint for establishing weak uniqueness in the next section.

Proposition 2. The process $\left(B_{t}^{\prime}\right)_{t \geq 0}$ is a standard Brownian motion which is independent of $\left(B_{t}\right)_{t \geq 0}$. Further, $W$ can be recovered from $\left(X, B, B^{\prime}\right)$ as

$$
W_{t}=B_{\tau(t)}+B_{\tau^{\prime}(t)}^{\prime},
$$

where

$$
\tau(t):=\inf \left\{s \geq 0: s+\sigma^{\prime}\left(A_{s}\right)>t\right\} \quad \text { and } \quad \tau^{\prime}(t):=t-\tau(t) .
$$

Proof. That $\left(B_{t}^{\prime}\right)_{t \geq 0}$ is a Brownian motion which is independent of $\left(B_{t}\right)_{t \geq 0}$ follows immediately from the definition of $B$ and $B^{\prime}$ and Knight's extension of the Dambis-DubinsSchwarz theorem (see e.g. [12, p. 183]).

Then we simply write

$$
W_{t}=\int_{0}^{t} \mathbf{1}_{\left\{Y_{s}>I_{s}\right\}} d W_{s}+\int_{0}^{t} \mathbf{1}_{\left\{Y_{s}=I_{s}\right\}} d W_{s}=B_{\tau(t)}+B_{\tau^{\prime}(t)}^{\prime},
$$

where

$$
\tau(t):=\int_{0}^{t} \mathbf{1}_{\left\{Y_{s}>I_{s}\right\}} d s \text { and } \tau^{\prime}(t):=\int_{0}^{t} \mathbf{1}_{\left\{Y_{s}=I_{s}\right\}} d s .
$$

The identity $\tau(t)+\tau^{\prime}(t)=t$ is obvious. By definition, $T_{t}=\inf \{s \geq 0: \tau(s)>t\}$ and thus $\tau(\cdot)$ coincides with the continuous left inverse of the strictly increasing time-change $T$. i.e. $\tau\left(T_{t}\right) \equiv t$. We infer from Lemma 1 that $\tau(t)=\inf \left\{s \geq 0: s+\sigma^{\prime}\left(A_{s}\right)>t\right\}$.

Remark. By a more careful analysis, one could also establish a stronger result of independence, namely that $X$ and $B^{\prime}$ are independent processes. Nonetheless, as this will not be needed in this work and also follows from the analysis in the next section, we leave the direct proof to the interested reader. In this connection, we also stress that the Brownian motion $B$ is adapted to the natural filtration of $X$, as one sees from Proposition 1 . But we do not know whether, conversely, $X$ is adapted to the natural filtration of the Brownian motion $B$, that is, whether the solution to 4 is strong. 


\section{Uniqueness in distribution}

In this section, we consider some filtered probability space $\left(\Omega,\left(\mathcal{F}_{t}\right)_{t \geq 0}, \mathbb{P}\right)$. We assume there is an $\left(\mathcal{F}_{t}\right)$-Brownian motion $\left(B_{t}\right)_{t \geq 0}$ and an adapted process $\left(X_{t}\right)_{t \geq 0}$ which satisfies equations (1) and (4) and starts with $X_{0}=\dot{X}_{0}=0$. Our goal is to establish that $\left(X_{t}\right)_{t \geq 0}$ has the distribution of the process of the preceding section. In this direction, Proposition 2 points at the role of an independent Brownian motion, so we assume that the same probability space $\Omega$ can be endowed with another filtration $\left(\mathcal{F}_{t}^{\prime}\right)_{t \geq 0}$ such that the terminal $\sigma$-fields $\mathcal{F}_{\infty}$ and $\mathcal{F}_{\infty}^{\prime}$ are independent, and that there exists an $\left(\mathcal{F}_{t}^{\prime}\right)$-Brownian motion $\left(B_{t}^{\prime}\right)_{t \geq 0}$. Clearly, these assumptions involve no loss of generality (as it suffices to enlarge the initial probability space).

Just as in the preceding section, we then write

$$
\sigma^{\prime}(x):=\inf \left\{t \geq 0: B_{t}^{\prime}>x\right\}
$$

for the first passage time of $B^{\prime}$ above level $x \geq 0$, and define

$$
T_{t}:=t+\sigma^{\prime}\left(A_{t}\right), \quad t \geq 0 .
$$

The process $t \mapsto T_{t}$ is strictly increasing and thus has a continuous left inverse

$$
\tau(t):=\inf \left\{s \geq 0: s+\sigma^{\prime}\left(A_{s}\right)>t\right\}, \quad t \geq 0,
$$

i.e. $\tau\left(T_{t}\right) \equiv t$. Clearly $0 \leq \tau(t) \leq t$, and we also set

$$
\tau^{\prime}(t):=t-\tau(t), \quad t \geq 0 .
$$

Finally, we define

$$
W_{t}:=B_{\tau(t)}+B_{\tau^{\prime}(t)}^{\prime}, \quad t \geq 0 .
$$

The weak uniqueness stated in Theorem 2 (ii) is now a consequence of the following.

Proposition 3. (i) The process $\left(W_{t}\right)_{t \geq 0}$ is a standard Brownian motion.

(ii) The process $\left(X_{t}\right)_{t \geq 0}$ can be recovered as

$$
X_{t}=(Y-I) \circ T_{t}, \quad t \geq 0,
$$

where

$$
Y_{t}:=\int_{0}^{t} W_{s} d s \text { and } I_{t}:=\inf \left\{Y_{s}: 0 \leq s \leq t\right\} .
$$

(iii) Finally, we have the identity

$$
T_{t}=\inf \left\{s \geq 0: \int_{0}^{s} \mathbf{1}_{\left\{Y_{v}>I_{v}\right\}} d v>t\right\}, \quad t \geq 0 .
$$


Remark. Proposition 3 shows that $X$ is distributed as the process which appears in Theorem 1 and as a consequence, we must have $\int_{0}^{\infty} \mathbf{1}_{\left\{X_{t}=0\right\}} d t=0$ a.s. It may be interesting to point out that this property can be checked directly from (4) and (1). More precisely, the set of times $t$ when $X_{t}=0$ is contained in the zero set of the Brownian semi-martingale $\dot{X}=B+A$. That the latter has zero Lebesgue measure a.s. can be seen from the occupation density formula for Brownian semi-martingales (see e.g. Corollary 1 [11, p. 216]).

The rest of this section is devoted to the proof of Proposition 3, we start with the first part.

Proof of Proposition $3(i)$. Although one can perhaps establish the result more directly by stochastic calculus, we shall use an approximation, as this makes the proof more intuitive. Specifically, pick $\varepsilon>0$ and introduce

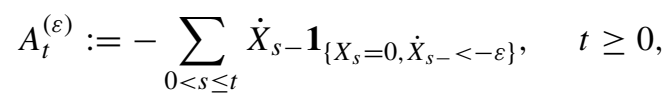

so that $t \mapsto A_{t}^{(\varepsilon)}$ is a nondecreasing process and

$$
\lim _{\varepsilon \downarrow 0} \uparrow A_{t}^{(\varepsilon)}=A_{t}, \quad t \geq 0 .
$$

Set also

$$
\tau^{(\varepsilon)}(t):=\inf \left\{s \geq 0: s+\sigma^{\prime}\left(A_{s}^{(\varepsilon)}\right)>t\right\},
$$

so $t \mapsto \tau^{(\varepsilon)}(t)$ is a continuous nondecreasing process with $0 \leq \tau^{(\varepsilon)}(t) \leq t$ and

$$
\lim _{\varepsilon \downarrow 0} \downarrow \tau^{(\varepsilon)}(t)=\tau(t), \quad t \geq 0 .
$$

Thus, if we define

$$
W_{t}^{(\varepsilon)}:=B_{\tau^{(\varepsilon)}(t)}+B_{t-\tau^{(\varepsilon)}(t)}^{\prime}, \quad t \geq 0
$$

then we have

$$
\lim _{\varepsilon \rightarrow 0+} W_{t}^{(\varepsilon)}=W_{t}
$$

Hence, it now suffices to check that for every $\varepsilon>0$, the process $\left(W_{t}^{(\varepsilon)}\right)_{t \geq 0}$ is a standard Brownian motion. Let us first provide an intuitive explanation. The time-change $t \mapsto \tau^{(\varepsilon)}(t)$ is an absolutely continuous process, and its derivative $d \tau^{(\varepsilon)}(t) / d t:=\dot{\tau}^{(\varepsilon)}(t)$ is a step process which takes alternately the values 1 and 0 . The dual time-change $t \mapsto$ $t-\tau^{(\varepsilon)}(t)$ is also absolutely continuous with derivative $1-\dot{\tau}^{(\varepsilon)}(t) \in\{0,1\}$. The process $W^{(\varepsilon)}$ is thus obtained by following alternately the paths of two independent Brownian motions, $B$ and $B^{\prime}$, in a way which may remind us of the classical two-arm bandit (see, e.g., [4]). More precisely, $W^{(\varepsilon)}$ follows $B$ when $\dot{\tau}^{(\varepsilon)}(t)=1$ and follows $B^{\prime}$ otherwise. The instants when $W^{(\varepsilon)}$ switches from $B$ to $B^{\prime}$ correspond to the jump times of $A^{(\varepsilon)}$, whereas the instants when $W^{(\varepsilon)}$ switches from $B^{\prime}$ to $B$ correspond to certain first passage times of $B^{\prime}$. These switching times form an increasing sequence of predictable random times for $W^{(\varepsilon)}$, and we can then deduce from the strong Markov property that $W^{(\varepsilon)}$ is a standard Brownian motion. 
More precisely, the assumption that $X$ solves (4) and elementary properties of the free Langevin process easily imply that with probability 1 , the set of times $t$ at which $X_{t}$ hits the boundary 0 with incoming velocity $\dot{X}_{t-}<-\varepsilon$ is both discrete and unbounded. Thus the set of jump times of $A^{(\varepsilon)}$ can be expressed as an increasing sequence of stopping times $J_{1}^{(\varepsilon)}<J_{2}^{(\varepsilon)}<\cdots$ where $J_{0}^{(\varepsilon)}=0$ and

$$
J_{n}^{(\varepsilon)}:=\inf \left\{t>J_{n-1}^{(\varepsilon)}: X_{t}=0 \text { and } \dot{X}_{t-}<-\varepsilon\right\}, \quad n \in \mathbb{N},
$$

and $\lim _{n \rightarrow \infty} J_{n}^{(\varepsilon)}=\infty$.

Write for simplicity $a_{n}^{(\varepsilon)}=A_{J_{n}^{(\varepsilon)}}^{(\varepsilon)}$, and consider the increasing sequence $\left(\sigma^{\prime}\left(a_{n}^{(\varepsilon)}\right)\right)_{n \in \mathbb{N}}$. As each $a_{n}^{(\varepsilon)}$ is a random variable which is measurable with respect to $\mathcal{F}_{\infty}$ and thus independent of $B^{\prime}$, the $\sigma^{\prime}\left(a_{n}^{(\varepsilon)}\right)$ form an increasing sequence of randomized $\left(\mathcal{F}_{t}^{\prime}\right)$-stopping times. The strong Markov property entails that conditionally on $\left(a_{n}^{(\varepsilon)}\right)_{n \in \mathbb{N}}$, the pieces of Brownian paths

$$
\left(B_{t+\sigma^{\prime}\left(a_{n-1}^{(\varepsilon)}\right)}^{\prime}-a_{n-1}^{(\varepsilon)}: 0 \leq t<\sigma^{\prime}\left(a_{n}^{(\varepsilon)}\right)-\sigma^{\prime}\left(a_{n-1}^{(\varepsilon)}\right)\right)
$$

are independent, and for each fixed $n \in \mathbb{N}$, the conditional law of this $n$-th piece is that of a standard Brownian motion killed when it exceeds $a_{n}^{(\varepsilon)}-a_{n-1}^{(\varepsilon)}$.

The process $\left(W_{t}^{(\varepsilon)}\right)_{t \geq 0}$ is obtained by splicing the sequence of pieces of paths

$\left(B_{t}: 0 \leq t<J_{1}^{(\varepsilon)}\right),\left(B_{t}^{\prime}: 0 \leq t<\sigma^{\prime}\left(a_{1}^{(\varepsilon)}\right)\right),\left(B_{t+J_{1}^{(\varepsilon)}}-B_{J_{1}^{(\varepsilon)}}: 0 \leq t<J_{2}^{(\varepsilon)}-J_{1}^{(\varepsilon)}\right), \ldots$ In particular

$$
W_{t}^{(\varepsilon)}= \begin{cases}B_{t} & \text { when } 0 \leq t<J_{1}^{(\varepsilon)}, \\ B_{t-J_{1}^{\prime}(\varepsilon)}^{\prime}+B_{J_{1}^{(\varepsilon)}} & \text { when } J_{1}^{(\varepsilon)} \leq t<J_{1}^{(\varepsilon)}+\sigma^{\prime}\left(a_{1}^{(\varepsilon)}\right),\end{cases}
$$

and the strong Markov property (relative to the filtration $\left(\mathcal{F}_{t}\right)$ ) of Brownian motion shows that the process $\left(W_{t}^{(\varepsilon)}: 0 \leq t<J_{1}^{(\varepsilon)}+\sigma^{\prime}\left(a_{1}^{(\varepsilon)}\right)\right)$ has the same law as $\left(B_{t}: 0 \leq t\right.$ $\left.<\rho_{1}\right)$ where $\rho_{1}$ is the $\left(\mathcal{F}_{t}\right)$-stopping time defined as $\rho_{1}:=\inf \left\{t>J_{1}^{(\varepsilon)}: B_{t}-B_{J_{1}^{(\varepsilon)}}\right.$ $>a_{1}^{(\varepsilon)}$. Splicing more and more pieces, we now see by an iteration of this argument that $\left(W_{t}^{(\varepsilon)}: 0 \leq t<J_{n}^{(\varepsilon)}+\sigma^{\prime}\left(a_{n}^{(\varepsilon)}\right)\right)$ has the same distribution as $\left(B_{t}: 0 \leq t<\rho_{n}\right)$ where $\rho_{n}:=\inf \left\{t>J_{n}^{(\varepsilon)}: B_{t}-B_{J_{n}^{(\varepsilon)}}>a_{n}^{(\varepsilon)}\right\}$. Letting $n \rightarrow \infty$, we conclude that $\left(W_{t}^{(\varepsilon)}\right)_{t \geq 0}$ is a standard Brownian motion, and an appeal to (6) completes the proof.

Next, let us write

$$
\mathcal{D}_{A}:=\left\{t>0: A_{t}>A_{t-}\right\}
$$

for the set of times where $A$ is discontinuous. Observe from (4) that we also have the identification

$$
\mathcal{D}_{A}=\left\{t>0: X_{t}=0 \text { and } \dot{X}_{t-}<0\right\}
$$

as the set of instants when $X$ hits the boundary 0 with a strictly negative incoming velocity. An important step in the proof of Proposition 3 is provided by the following representation of the processes $\tau(\cdot)$ and $\tau^{\prime}(\cdot)$. 
Lemma 2. Introduce the random open set

$$
\left.\mathcal{O}:=\bigcup_{t \in \mathcal{D}_{A}}\right] t+\sigma^{\prime}\left(A_{t-}\right), t+\sigma^{\prime}\left(A_{t}\right)[,
$$

and write $\mathcal{O}^{c}:=[0, \infty[\backslash \mathcal{O}$ for its complement.

(i) The processes $t \mapsto \tau(t)$ and $t \mapsto \tau^{\prime}(t)$ are both absolutely continuous nondecreasing processes with Stieltjes measures given by

$$
d \tau(t)=\mathbf{1}_{\mathcal{O}^{c}} d t, \quad d \tau^{\prime}(t)=\mathbf{1}_{\mathcal{O}} d t .
$$

(ii) We have $W_{t} \leq 0$ and $X_{\tau(t)}=0$ for every $t \in \mathcal{O}$, a.s.

Proof. (i) Write $\lambda$ for the Lebesgue measure on $\mathbb{R}_{+}$. We have to show that

$$
\lambda(\mathcal{O} \cap[0, t])=\tau^{\prime}(t), \quad t \geq 0 .
$$

To do this, let $\mathcal{A}$ denote the closed range of the process $A$., i.e. the set of points of the type $A_{t}$ or $A_{t-}$ for some $t \geq 0$. The complement $\mathcal{A}^{c}:=\mathbb{R}_{+} \backslash \mathcal{A}$ has a canonical decomposition as union of disjoint open intervals, given by

$$
\left.\mathcal{A}^{c}=\bigcup_{t \in \mathcal{D}_{A}}\right] A_{t-}, A_{t}[.
$$

Observe that, since $A$. is a pure jump process, for every $t \geq 0$ we have

$$
\lambda\left(\left[0, A_{t}\right] \backslash \mathcal{A}\right)=\sum_{s \in \mathcal{D}_{A} \cap[0, t]}\left(A_{s}-A_{s-}\right)=A_{t},
$$

and hence $\lambda(\mathcal{A})=0$.

On the other hand, it is well-known that the first passage process $\sigma^{\prime}$ is a stable subordinator with index $1 / 2$. In particular, it is purely discontinuous, and, by the Lévy-Itô decomposition, the process of its jumps is a Poisson point process. Since $\mathcal{A}$ has zero Lebesgue measure and is independent of $\sigma^{\prime}, \mathcal{A}$ does not contain any jump time of $\sigma^{\prime}$, a.s. It follows that for every $v \geq 0$,

$$
\lambda\left(\mathcal{O} \cap\left[0, v+\sigma^{\prime}\left(A_{v}\right)\right]\right)=\sum_{s \in \mathcal{D}_{A} \cap[0, v]}\left(\sigma^{\prime}\left(A_{s}\right)-\sigma^{\prime}\left(A_{s-}\right)\right)=\sigma^{\prime}\left(A_{v}\right) .
$$

Recall now that $T_{v}=v+\sigma^{\prime}\left(A_{v}\right)$ and observe that

$$
\tau^{\prime}\left(T_{v}\right)=T_{v}-\tau\left(T_{v}\right)=T_{v}-v=v+\sigma^{\prime}\left(A_{v}\right)-v=\sigma^{\prime}\left(A_{v}\right) .
$$

We have thus checked that (7) holds for every $t$ of the form $t=T_{v}$ for some $v \geq 0$, and hence, by approximation, also for every $t$ of the form $t=T_{v-}$ for some $v>0$.

Finally, suppose that $t \in] T_{v-}, T_{v}$ [, where $v=\tau(t)$. From the above we get

$$
\lambda(\mathcal{O} \cap[0, t])=\lambda\left(\mathcal{O} \cap\left[0, T_{v-}\right]\right)+t-T_{v-}=\tau^{\prime}\left(T_{v-}\right)+t-T_{v-} .
$$


But

$$
\tau^{\prime}\left(T_{v-}\right)+t-T_{v-}=T_{v-}-\tau\left(T_{v-}\right)+t-T_{v-}=t-v=t-\tau(t)=\tau^{\prime}(t),
$$

and we conclude that (7) holds for all $t \geq 0$.

(ii) If $t \in \mathcal{O}$, then $t \in] T_{v-}, T_{v}$ [ where $v=\tau(t)$. By definition,

$$
W_{t}=B_{v}+B_{t-v}^{\prime} .
$$

On the other hand, $v \in \mathcal{D}_{A}$ and thus $X_{v}=0$. Further, by (4) and (1), we have $\dot{X}_{v}=$ $B_{v}+A_{v}=0$. We deduce that

$$
W_{t}=B_{t-v}^{\prime}-A_{v} \leq 0,
$$

as $t-v<\sigma^{\prime}\left(A_{v}\right)$ (because $t<T_{v}=v+\sigma^{\prime}\left(A_{v}\right)$ ).

We are now able to establish the second part of Proposition 3

Proof of Proposition 3 ii). We decompose

$$
Y_{t}=\int_{0}^{t} W_{s} d s=\int_{0}^{t} W_{s} d \tau(s)+\int_{0}^{t} W_{s} d \tau^{\prime}(s) .
$$

The change of variables $s=T_{v}$ enables us to rewrite the first integral on the right-hand side as

$$
\int_{0}^{t} W_{s} d \tau(s)=\int_{0}^{\tau(t)} W_{T_{v}} d v=\int_{0}^{\tau(t)}\left(B_{\tau\left(T_{v}\right)}+B_{T_{v}-\tau\left(T_{v}\right)}^{\prime}\right) d v .
$$

Since $\tau\left(T_{v}\right)=v$ and $T_{v}-\tau\left(T_{v}\right)=T_{v}-v=\sigma^{\prime}\left(A_{v}\right)$, the right-hand side equals

$$
\int_{0}^{\tau(t)}\left(B_{v}+A_{v}\right) d v=\int_{0}^{\tau(t)} \dot{X}_{v} d v=X_{\tau(t)},
$$

where the first equality follows from (4).

Next, we write $I_{t}^{\prime}:=\int_{0}^{t} W_{s} d \tau^{\prime}(s)$, so that the process $t \mapsto Y_{t}-I_{t}^{\prime}=X_{\tau(t)}$ is nonnegative. Further, we know from Lemma 2(ii) that $t \mapsto I_{t}^{\prime}$ is a nonincreasing process and that the Stieltjes measure $d\left(-I_{t}^{\prime}\right)$ assigns no mass to $\mathcal{O}^{c}$, and a fortiori is supported on the set of times $t$ such that $Y_{t}-I_{t}^{\prime}=X_{\tau(t)}=0$. An application of Skorokhod's reflection principle (see for instance [12, p. 239]) enables us to make the identification $I_{t}^{\prime}=\inf \left\{Y_{s}: 0 \leq s \leq t\right\}=I_{t}$. We conclude that $X_{t}=X_{\tau\left(T_{t}\right)}=Y_{T_{t}}-I_{T_{t}}$.

Finally, we turn our attention to the third part of Proposition 3.

Proof of Proposition 3 iii). On the one hand, we have seen in the proof of part (ii) above that the infimum $I$ of the free Langevin process $Y$ can be expressed as

$$
I_{t}=\int_{0}^{t} W_{s} d \tau^{\prime}(s)=\int_{0}^{t} \mathbf{1}_{\mathcal{O}}(s) W_{s} d s,
$$

where the second identity follows from Lemma 2 . 
On the other hand, we must have $W_{t} \leq 0$ for every $t$ such that $Y_{t}=I_{t}$. Indeed, if we had $W_{t}>0$ for such a time $t$, then $Y$ would be strictly increasing on some neighborhood of $t$, which is absurd. Now for every $t \geq 0$ such that $Y_{t}=I_{t}$ and $W_{t}<0, Y$ is strictly decreasing on some interval $\left[t, t^{\prime}\right]$ with $t^{\prime}>t$, and thus $Y=I$ on $\left[t, t^{\prime}\right]$. Since the total time that $W$ spends at 0 is zero, we also obtain

$$
I_{t}=\int_{0}^{t} \mathbf{1}_{\left\{Y_{s}=I_{s}\right\}} W_{s} d s .
$$

Using again the fact that the total time that $W$ spends at 0 is zero, we deduce by comparison of these two expressions that with probability one, the random sets $\mathcal{O}$ and $\left\{s \geq 0: Y_{s}=I_{s}\right\}$ coincide $\lambda$-almost everywhere. More precisely, recall the notation $\mathcal{I}:=\left\{s \geq 0: Y_{s}=I_{s}\right\}$ and that the boundary $\partial \mathcal{I}=\mathcal{I} \backslash \mathcal{I}^{\circ}$ has zero Lebesgue measure. We now see that the open sets $\mathcal{O}$ and $\mathcal{I}^{\circ}$ coincide a.s. As a consequence of Lemma 2 .

$$
\tau(t)=\int_{0}^{t} \mathbf{1}_{\mathcal{O}^{c}}(s) d s=\int_{0}^{t} \mathbf{1}_{\left\{Y_{s}>I_{s}\right\}} d s, \quad t \geq 0,
$$

and since $T$. is the right-continuous inverse of $\tau(\cdot)$, this completes the proof.

\section{Some comments and questions}

We mentioned in the Introduction that second order differential equations with constraints of the type (1) and (2) may have multiple solutions even in the situation when the external force $F$ is smooth. This was first pointed out by Bressan [3], who also conjectured that uniqueness holds when the force is a polynomial function of time. Schatzman [13] formulated the general setting of second order differential inclusions, and established a general theorem on existence of solutions. She also recovered independently Bressan's example of a force of class $\mathcal{C}^{\infty}$ for which such a system has multiple solutions. Percivale [10] was the first to show that uniqueness holds for systems with only one degree of freedom, when the force is given by an analytic function of time and depends neither on the position nor on the velocity of the particle, and then Schatzman [14] extended this to the much harder case when the force is an analytic function of time, position and velocity. Finally, Ballard [1] considered more general discrete systems with several degrees of freedom and established that uniqueness always holds in the case when the force is analytic. We also refer to Maury [7, 8], Moreau [9] and Stewart [15] for numerical schemes for the computation of the motion of many-body systems with inelastic impacts.

For the convenience of the reader, we give a simple example of an external force of class $\mathcal{C}^{k}$ (for any fixed $k \in \mathbb{N}$ ) for which multiple solutions to (1) and (2) exist. Consider the increasing sequences $0<\cdots<s_{n}<t_{n}<s_{n+1}<\cdots$ given by

$$
s_{n}:=2^{2 n} \quad \text { and } \quad t_{n}:=2^{2 n+1}, \quad n \in \mathbb{Z} .
$$

Then introduce the convex increasing function $\alpha:[0, \infty[\rightarrow[0, \infty[$ which is linear on the intervals $\left[s_{n}, s_{n+1}\right]$ and such that $\alpha\left(s_{n}\right)=s_{n}^{k+3}$. Similarly, let $\beta:[0, \infty[\rightarrow[0, \infty[$ 
denote the convex increasing function which is linear on the intervals $\left[t_{n}, t_{n+1}\right]$ and such that $\beta\left(t_{n}\right)=t_{n}^{k+3}$. Observe that $\alpha$ and $\beta$ enjoy a property of self-similarity, namely

$$
\alpha(4 u)=4^{k+3} \alpha(u) \quad \text { and } \quad \beta(4 u)=4^{k+3} \beta(u), \quad u>0 .
$$

It should be obvious from a picture that there exists a function $\varphi:] 0, \infty[\rightarrow \mathbb{R}$ of class $\mathcal{C}^{k+3}$, which is bounded from above by both $\alpha$ and $\beta$, enjoys the same property of selfsimilarity, i.e. $\varphi(4 u)=4^{k+3} \varphi(u)$, and satisfies the following requirements:

$$
\left\{\begin{array}{l}
\varphi(u)=\alpha(u) \Leftrightarrow u=s_{n} \text { for some } n \in \mathbb{Z}, \\
\varphi(u)=\beta(u) \Leftrightarrow u=t_{n} \text { for some } n \in \mathbb{Z}, \\
\dot{\varphi}\left(s_{n}\right)=\dot{\alpha}\left(s_{n}+\right) \text { for every } n \in \mathbb{Z}, \\
\dot{\varphi}\left(t_{n}\right)=\dot{\beta}\left(t_{n}+\right) \text { for every } n \in \mathbb{Z} .
\end{array}\right.
$$

More precisely, one constructs first a function $\varphi$ which satisfies the preceding requirements on the interval $[1,4]$, in such a way that for every $l=0, \ldots, k+3$, the $l$-th derivative $\varphi^{(l)}$ of $\varphi$ has $\varphi^{(l)}(4-)=4^{k+3-l} \varphi^{(l)}(1+)$. Then $\varphi$ is extended to $] 0, \infty[$ by self-similarity, and we set $\varphi(0)=0$. Again by self-similarity, $\varphi$ is now of class $\mathcal{C}^{k+2}$ on $\left[0, \infty\left[\right.\right.$ with $\varphi^{(l)}(0+)=0$ for every $l=0, \ldots, k+2$. The requirements imply that $X_{u}:=\alpha(u)-\varphi(u)$ solves (1) and (2) with $F_{u}:=-\ddot{\varphi}(u)$ and $A_{u}:=\dot{\alpha}(u)$. Similarly, $X_{u}:=\beta(u)-\varphi(u)$ solves (1) and (2) with the same external force and $A_{u}:=\dot{\beta}(u)$. Hence equations (1) and (2) have at least two distinct solutions for $F_{u}:=-\ddot{\varphi}(u)$.

Self-similarity is merely used above as a convenient tool for checking the regularity of the external force at 0 , and a perusal of the argument reveals that a large class of counterexamples to uniqueness can be built by mimicking the preceding construction, using now an arbitrary strictly convex increasing function $c:\left[0, \infty\left[\rightarrow\left[0, \infty\left[\left(c(u)=u^{k+3}\right.\right.\right.\right.\right.$ in the example above), and arbitrary increasing sequences $\left(s_{n}\right)_{n \in \mathbb{Z}}$ and $\left(t_{n}\right)_{n \in \mathbb{Z}}$ with no common point and such that $\lim _{n \rightarrow-\infty} s_{n}=\lim _{n \rightarrow-\infty} t_{n}=0$. The external force $F=-\ddot{\varphi}$ may then no longer be smooth; note that in any case, $F$ has strong oscillations near zero, in the sense that $F$ takes negative and positive values at times arbitrarily close to 0 . This may suggest that, informally, existence of multiple solutions to (1) and (2) could hold for quite general external forces $F$ with strong oscillations. In this direction, recall that uniqueness of the solution has only been established for analytic external forces (see Ballard [1]).

Theorem 2 is thus in sharp contrast with the preceding observations, even though the uniqueness is only stated there in a weak sense. Hence an important open question is to ask whether pathwise uniqueness holds for equations (1) and (4).

Another interesting problem in this vein is to decide whether or not the solution which has been constructed in Section 2 is adapted to the natural filtration of the Brownian motion $\left(B_{t}\right)_{t \geq 0}$. One says that the solution is strong if the answer is positive. We refer to Tsirel'son [16] for a classical example of an SDE which has a unique weak solution, but no strong solution.

Acknowledgments. I would like to thank Patrick Ballard and Bertrand Maury for useful historical comments and references about the problem which motivated this work. 


\section{References}

[1] Ballard, P.: The dynamics of discrete mechanical systems with perfect unilateral constraints. Arch. Ration. Mech. Anal. 154, 199-274 (2000) Zbl 0965.70024 MR 1785473

[2] Bertoin, J.: Reflecting a Langevin process at an absorbing boundary. Ann. Probab. 35, 20212037 (2007) Zbl pre05211864 MR 2353380

[3] Bressan, A.: Incompatibilità dei teoremi di esistenza e di unicità del moto per un tipo molto comune e regolare di sistemi meccanici. Ann. Scuola Norm. Sup. Pisa (3) 14, 333-348 (1960) Zbl 0096.38204 MR 0133950

[4] Gittins, J. C.: Multi-Armed Bandit Allocation Indices. Wiley-Interscience Series in Systems and Optimization, Wiley, Chichester (1989) Zbl 0699.90068 MR 0996417

[5] Lachal, A.: Applications de la théorie des excursions à l'intégrale du mouvement brownien. Séminaire de Probabilités XXXVIII, Lecture Notes in Math. 1801, Springer, 109-195 (2003) Zbl 1045.60084 MR 2053045

[6] Lapeyre, B.: Une application de la théorie des excursions à une diffusion réfléchie dégénérée. Probab. Theory Related Fields 87, 189-207 (1990) Zbl 0694.60052 MR 1080489

[7] Maury, B.: Direct simulation of aggregation phenomena. Comm. Math. Sci. 2, suppl. 1, 1-11 (2004); http://intlpress.com/CMS/issueS-1/S-1.pdf Zbl 1086.76072 MR 2119870

[8] Maury, B.: A time-stepping scheme for inelastic collisions. Numer. Math. 102, 649-679 (2006) Zbl pre05014043 MR 2207284

[9] Moreau, J. J.: Some numerical methods in multibody dynamics: Application to granular materials. Eur. J. Mech. A 13, 93-114 (1994) Zbl 0815.73009 MR 1295862

[10] Percivale, D.: Uniqueness in the elastic bounce problem, I. J. Differential Equations 56, 206215 (1985) Zbl 0521.73006 MR 0774163

[11] Protter, Ph.: Stochastic Integration and Differential Equations. 2nd ed., Appl. Math. 21, Springer, Berlin (2004) Zbl 1041.60005 MR 2020294

[12] Revuz, D., Yor, M.: Continuous Martingales and Brownian Motion. 3rd ed., Grundlehren Math. Wiss. 293, Springer, Berlin (1999) Zbl 0917.60006 MR 1725357

[13] Schatzman, M.: A class of nonlinear differential equations of second order in time. Nonlinear Anal. 2, 355-373 (1978) Zbl 0382.34003 MR 0512664

[14] Schatzman, M.: Uniqueness and continuous dependence on data for one dimensional impact problems. Math. Comput. Modelling 28, 1-18 (1998) Zbl pre01737577 MR 1616372

[15] Stewart, D. E.: Convergence of a time-stepping scheme for rigid-body dynamics and resolution of Painlevé's problem. Arch. Ration. Mech. Anal. 145, 215-260 (1998) Zbl 0922.70004 MR 1664526

[16] Tsirel'son, B. S.: An example of a stochastic differential equation having no strong solution. Teor. Veroyatn. Primen. 20, 427-430 (1975) (in Russian); English transl.: Theory Probab. Appl. 20, 416-418 (1975) Zbl 0353.60061 MR 0375461 